
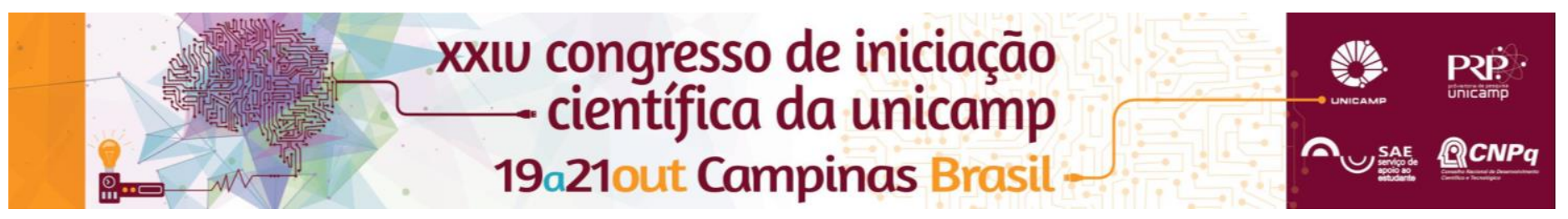

\title{
Introduction to Black Hole Information Paradox
}

\author{
Laura B. dos Penedos*, Pedro C. de Holanda
}

\begin{abstract}
We proposed an study of the black hole loss information starting from Thermodynamics and Black Hole Mechanics. The Bekenstein-Hawking entropy formula was derived and used to understand the implications of Hawking Radiation. Apart from this, we studied some concepts of singularity in General Relativity and Quantum gravity to understand the first model of loss information in black holes and why Black Hole Information Paradox is an unsolved problem in Physics at the present time.
\end{abstract}

\section{Key words:}

General Relativity, Black Hole, Information Paradox.

\section{Introduction}

Black Hole is formed by gravitational collapse and is described mathematically by an object consisted of a gravitational singularity which is covered by a trapped surface, the horizon event. The consequence is that no signals inside this surface can escape to spatial infinity, creating the term "black hole".

Because some results in General Relativity, namely, Penrose Process, the Hawking's theorem and Irreducible Mass, by Christodoulou, Hawking, Carter and Bardeen performed a derivation of the four laws of black hole mechanics making use of a thermodynamics analysis where black hole area is associated with entropy. This model is questioned when quantum effects are not negligible.

Furthermore, if we consider quantum phenomena it appears a thermal radiation emitted by black hole that is called Hawking Radiation. A new model imposes a Generalized Second Law for black hole mechanics and the black hole entropy converts to the Bekenstein-Hawking Entropy Formula.

The study of Black Hole Information Loss appears because Hawking Radiation makes the final quantum state cannot be described as a pure state, which creates a limitation to define the pure final state for the outside region. This phenomenon does not happen in the AdS/CFT (Anti-de Sitter space/Conformal Field Theory) correspondence which claims that there is no information loss. This is the Information Paradox and understand how it works is the primary objective of this research.

\section{Results and Discussion}

First of all, it was researched the theorems and conditions of singularity in General Relativity and how it applies to black holes. The No Hair Theorem and the Weak Energy Condidtion were found as the most important to calculations in formalism of black holes.

We studied the Penrose Process, Hawking's area theorem and the Irreducible Mass, formulated by Christodoulou. Each of them concluded that any transformation in black holes, particularly in irreversible processes, has a tendency to increase its area surface. Moreover, the Generalized Israel Conjecture states that the solution exterior to an absolute event horizon approaches a Kerr-Newmann solution in some conditions. These results make us to think intuitively that black hole could be associated with thermodynamics formalism. So, we use [1] to understand how this analysis can be done. The Generalized Smarr Law and the Four Laws of Black Hole Mechanics were derived.

This formalism works quite well in classical mechanics. But if we consider quantum effects, such as Hawking Radiation, the Second Law cannot describe the system. Find the Generalized Second Law was also a result of this research.

Following the steps in [2], we used a model of quantum gravity which is based on quantize action and, imposing Grand Canonical Ensemble to the partition function and making use of Smarr Formula, we derived the Bekenstein-Hawking Entropy Formula. This formula is directly proportional to event horizon surface area and inversely proportional to Planck constant. Find the meaning of Bekenstein-Hawking entropy formula is in process.

We studied the creation and annihilation operators applied in the formalism of Quantum Fields in curved spacetime to understand the primary idea of Hawking Radiation and why it relates with loss of information in black holes.

\section{Conclusions}

This work made an intensive bibliographic research, including a historical approach of each discovery. We also derived Smarr Formula, the Four Laws of Black Hole Mechanics and Bekenstein-Hawking Entropy Formula.

The next steps constitutes in understanding the meaning of Bekenstein-Hawking Entropy Formula and how it relates with information theory applied to black holes.

\section{Acknowledgement}

We would like to thank IFGW Library (BIF) for the excellent job bringing extra works from outside UNICAMP and SAEUNICAMP for the financial support.

${ }^{1}$ Bardeen, J.M.; Carter, B.; Hawking, S.W. Commun. Math. Phys. 31, 161-170 (1973).

${ }^{2}$ Gibbons, G.W.; Hawking, S.W. Phys. Rev. D 15, 10 (1977).

${ }^{3}$ Bekenstein, J.D. Phys. Rev. D 7, 8 (1973).

${ }^{4}$ Fabbri, A.; Navarro-Salas, J. Modeling Black Hole Evaporation (Imperial College Press, London, 2005). 